Article

\title{
Enhanced Osteogenic Differentiation of Human Primary Mesenchymal Stem and Progenitor Cultures on Graphene Oxide/Poly(methyl methacrylate) Composite Scaffolds
}

\author{
Katarzyna Krukiewicz ${ }^{1, *(\mathbb{D}}$, David Putzer ${ }^{2}{ }^{(}$, Nicole Stuendl ${ }^{3}$, Birgit Lohberger ${ }^{3}$ \\ and Firas Awaja 4,5,* \\ 1 Department of Physical Chemistry and Technology of Polymers, Silesian University of Technology, \\ 44-100 Gliwice, Poland \\ 2 Experimental Orthopedics, Department of Orthopedic Surgery, Medical University Innsbruck, \\ 6020 Innsbruck, Austria; david.putzer@i-med.ac.at \\ 3 Department of Orthopedics and Trauma, Medical University of Graz, 8036 Graz, Austria; \\ nicole.stuendl@medunigraz.at (N.S.); birgit.lohberger@medunigraz.at (B.L.) \\ 4 School of Medicine, National University of Ireland, H91 CF50 Galway, Ireland \\ 5 Engmat Ltd., Clybaun Road, H91 P96H Galway, Ireland \\ * Correspondence: katarzyna.krukiewicz@polsl.pl (K.K.); firas.awaja@gmail.com (F.A.)
}

Received: 4 June 2020; Accepted: 3 July 2020; Published: 5 July 2020

\begin{abstract}
Due to its versatility, small size, large surface area, and ability to interact with biological cells and tissues, graphene oxide (GO) is an excellent filler for various polymeric composites and is frequently used to expand their functionality. Even though the major advantage of the incorporation of GO is the enhancement of mechanical properties of the composite material, GO is also known to improve bioactivity during biomineralization and promote osteoblast adhesion. In this study, we described the fabrication of a composite bone cement made of GO and poly(methyl methacrylate) (PMMA), and we investigated its potential to enhance osteogenic differentiation of human primary mesenchymal stem and progenitor cells. Through the analysis of three differentiation markers, namely alkaline phosphatase, secreted protein acidic and rich in cysteine, and bone morphogenetic protein-2 in the presence and in the absence of an osteogenic differentiation medium, we were able to indicate a composite produced manually with a thick GO paper as the most effective among all investigated samples. This effect was related to its developed surface, possessing a significant number of voids and pores. In this way, GO/PMMA composites were shown as promising materials for the applications in bone tissue engineering.
\end{abstract}

Keywords: bone regeneration; graphene oxide; mesenchymal stem and progenitor cells; osteogenic differentiation; poly(methyl methacrylate)

\section{Introduction}

Since its discovery in 2004 [1], graphene has drawn immense attention of the scientific community and has become an object of intensive research. Due to its high planar surface area, superior mechanical strength, outstanding optical properties, as well as remarkable thermal and electrical conductivity [2-4], graphene has been widely used in a variety of applications, including transparent conductors, ultrafast transistors, precise biosensors, and tissue scaffolds [5]. The potential of graphene has been further expanded by introducing to its structure a variety of functional group, resulting in the fabrication of graphene oxide (GO). GO is usually produced by the oxidation of graphite, resulting in a partial 
breaking of $\mathrm{sp}^{2}$ bonds present in its structure and subsequent increase in the distance between carbon layers [3]. Therefore, GO possesses both hydrophobic (due to the presence of pristine graphite structure) and hydrophilic (due to the presence of hydroxyl, epoxy, carbonyl, and carboxyl groups) parts, and is characterized by affinity for aromatic rings, excellent aqueous processability, amphiphilicity, ease of functionalization, and biocompatibility [3,5]. Consequently, GO has been marked as an excellent material for numerous biomedical applications, including the design of biosensors [5], drug delivery systems [6], antimicrobial coatings [7], cell imaging platforms [8], and in gene therapy [9].

Due to its versatility, small size, large surface area, and ability to interact with biological cells and tissues, GO is an excellent filler for various polymers and is frequently used to expand their functionality. For instance, Wan et al. [10] reported an increase in the tensile strength, modulus, and energy at break, as well as the improvement in bioactivity during biomineralization simultaneously with maintaining high porosity when an electrospun poly( $\varepsilon$-caprolactone) membrane was reinforced with GO nanoplatelets. Also Baradaran et al. [11] observed the increase in elastic modulus and fracture toughness, as well as the promotion in osteoblast adhesion and proliferation when GO was used as a filler for hydroxyapatite nanosheets. On the other hand, calcium phosphate mineralized graphene oxide/chitosan scaffolds were found to express biomimicry, providing a suitable environment for cell adhesion and growth, and maintaining high mechanical strength [12]. GO was also demonstrated to act as an excellent filler for such polymer matrices as poly(vinyl alcohol) [13], poly(carbonate urethane) [14], hyaluronic acid [15], and poly(acrylic acid) [16], resulting in the formation of robust composite materials with applicability in biomedical engineering.

Poly(methyl methacrylate) (PMMA) is a non-toxic thermoplastic polymer possessing a very good toxicological safety record in biomedicine [17]. PMMA is frequently used as a screw fixation in bone, bone cement, filler for bone cavities and skull defects, as well as vertebrae stabilization in osteoporotic patients [18]. The exceptional applicability of PMMA in orthopedic and dental applications is caused by its good processability, handling properties, biocompatibility, suitable mechanical strength, and Young's modulus [19]. Despite its numerous merits, the common complications of using PMMA is bone resorption observed after implantation, which is the effect of the formation of a weak-link zone derived from not sufficient interactions between cement and a bone [20]. Therefore, the modification of PMMA has become a very active area of research. A promising way to improve the biological performance of PMMA is to blend it with an antibiotic, e.g., gentamycin [21]. This modification approach is nowadays a well established strategy that allows prevention of periprosthetic infections and osteomyelitis. Another way to enhance the performance of PMMA is to incorporate in its structure a filler with a particular functionality. For instance, loading of PMMA with multiwalled carbon nanotubes could significantly improve the mechanical properties and reduce the exothermic polymerization reaction of the bone cement [22]. The use of tri-calcium phosphate and chitosan as inorganic/organic additives to PMMA decreased polymer curing temperature, extended setting time, and increased weight loss and porosity after degradation and, among all, promoted better osteointegration than pure PMMA [23]. Also graphene and graphene oxide have been used as fillers to PMMA [24], improving the mechanical properties of PMMA, particularly its fracture toughness and fatigue performance. What was interesting, GO was found to outperform graphene and provide greater enhancements due to its high functionalization that increased the interfacial adhesion between a filler and PMMA matrix. Simultaneously, the presence of graphene or GO was not found to have any negative effect on the biocompatibility of PMMA composites, potentially allowing their further clinical progression [25].

In this paper, the potential of GO/PMMA composites for the application in bone tissue engineering is assessed by the analysis of three differentiation markers expressed by human primary mesenchymal stem and progenitor cells (hMSPCs) cultured on the top of the composites. By performing the cell culture in the presence and absence of a specific induction medium, we were able to determine the efficiency of osteogenic differentiation of hMSPCs cultured on four types of GO/PMMA scaffolds, differing in the thickness of GO paper as well as the method of fabrication of the composites. Microscopic 
analysis of the surface of materials allowed investigation of the biological behavior of the materials with respect to their surface morphology.

\section{Materials and Methods}

\subsection{Fabrication of $G O / P M M A$ Composites}

For the production of graphene oxide (GO) paper, $4 \mathrm{mg} / \mathrm{mL}$ suspension of GO flakes (GO monolayer content $>95 \%$, oxygen content between $40 \%$ and $50 \%$ ) in water was purchased from Graphenea (San Sebastián, Spain). GO suspension was inserted into Petri dishes with serological pipettes. The GO dispersion was dried in a shaking incubator with air fan for $48 \mathrm{~h}$ and inserted into an oven at $180^{\circ} \mathrm{C}$ for $20 \mathrm{~min}$. By changing the volume of GO suspension, two types of GO paper were fabricated, i.e., thin ( $8 \mathrm{~mL}$ of $\mathrm{GO}$ suspension) and thick (10 $\mathrm{mL}$ of $\mathrm{GO}$ suspension), designated as $\mathrm{GO}_{(\mathrm{A})}$ paper and $\mathrm{GO}_{(\mathrm{B})}$ paper, respectively.

For the fabrication of GO/PMMA composites, GO paper as well as SIMPLEX P (Stryker, Kalamazoo, MI, USA) radiopaque bone cement (prepared according to the manufacturer's instructions with a full dose of liquid monomer) were applied. The monomer was mixed to the polymer manually under laboratory conditions. The PMMA cement was then inserted in a metal casting form and covered with GO paper. The bone cement was kept in place for $30 \mathrm{~min}$ to guarantee sufficient hardening. Screws were closed after $15 \mathrm{~min}$. Two different methods were used to prepare combined samples including GO paper and bone cement (Scheme 1).

A

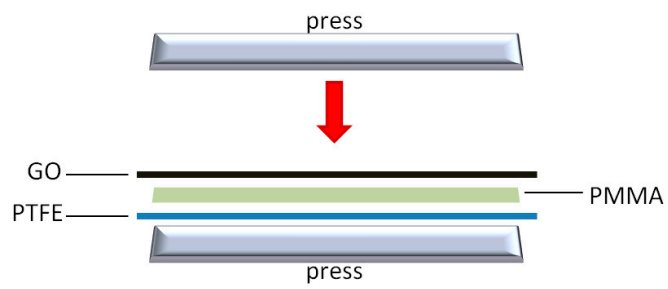

B

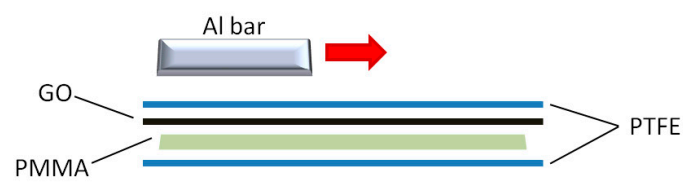

Scheme 1. Schematic representation of a fabrication process of GO/PMMA $(\mathrm{P})(A)$ and $\mathrm{GO} / \mathrm{PMMA}_{(\mathrm{M})}(\mathbf{B})$ composite materials.

In the first method, GO paper was placed on the bottom of a steel flat press (covered with poly(tetrafluoroethylene), PTFE, sheet to simplify cement detachment). The cement was spread on the upper PTFE sheet and then placed in contact with the GO paper. Then, the press was closed after $15 \mathrm{~min}$ to reach minimum thickness until the cement was polymerized ( $30 \mathrm{~min})$. The samples prepared in this way were designated as GO/PMMA $(\mathrm{P})$. In the second method, a compound material was produced manually: GO paper was laid down on a PTFE sheet, and then the cement was spread on the upper PTFE sheet and then placed in contact with the GO paper. An aluminum bar was used to spread the cement on GO paper within the two PTFE sheets. In this case, spreading the cement was more difficult, and led to the formation of a non-homogeneous PMMA layer with GO paper broken up into little pieces, which may have been due to the shrinking and expanding behavior of PMMA during the cement hardening phase. The samples prepared in this way were designated as GO/PMMA $(\mathrm{M})$.

Consequently, four types of samples were analyzed: $\mathrm{GO}_{(\mathrm{A})} / \mathrm{PMMA}_{(\mathrm{P})}, \mathrm{GO}_{(\mathrm{B})} / \mathrm{PMMA}_{(\mathrm{P})}$, $\mathrm{GO}_{(\mathrm{A})} / \mathrm{PMMA}_{(\mathrm{M})}$, and $\mathrm{GO}_{(\mathrm{B})} / \mathrm{PMMA}_{(\mathrm{M})}$, all with PMMA as the surface layer. For the scanning electron microscopic (SEM) investigations, a FEI Quanta 250 field emission gun (Thermo Fisher Scientific, Hillsboro, OR, USA) was used under high vacuum conditions and $20 \mathrm{kV}$ high tension. The micrographs were recorded with the Everhart-Thornley-Detector in secondary electron (SE) 
mode. The surfaces were sputter coated with a $10 \mathrm{~nm}$ thin layer of gold in order to provide sufficient electrical conductivity.

\subsection{Tissue Harvest and Cell Culture}

Explant hMSPCs were established from tissue samples of spongiosa bone harvested during routine hip joint surgeries. The study protocol was approved by the local ethics committee (reference number 29-156ex16/17), and informed consent was obtained from each orthopedic surgery patient. The study included a total of three patients, aged between 25 and 35, excluding pregnant women and those suffering from local inflammatory processes, metabolic bone diseases, and impaired blood coagulation. The length of harvested bone samples was kept between 4 and $6 \mathrm{~mm}$, and showed either cortical or cortical and cancellous structure. The samples were extensively rinsed with a phosphate-buffered saline (PBS; PAA Laboratory, Pasching, Austria) and transferred into $75 \mathrm{~cm}^{2}$ culture flasks (TPP, Trasadingen, Switzerland) with an appropriate volume of culture medium. For cell isolation and expansion, the samples were incubated in a humidified atmosphere $\left(5 \% \mathrm{CO}_{2}, 37^{\circ} \mathrm{C}\right)$.

\subsection{Flow Cytometry}

For a flow cytometric analysis, a total of $1 \times 10^{5}$ hMSPCs were resuspended in $200 \mu \mathrm{L}$ PBS. The characterization of cells was achieved with the use of commercial monoclonal antibodies, namely CD73 PE, CD90 APC, CD105 PE, CD45 APC-Cy7, CD34 APC, CD14 FITC, CD19 APC, and HLA-DR APC (BD Bioscience, San Jose, CA, USA). Titration had previously been used to determine the optimal amount of each antibody. Subsequently, two-color staining panels were used to present a combination of antibodies with non overlapping spectra. Negative cell lines and matched fluorochrome-conjugated isotype controls were applied to perform a background staining for antibodies. FACS LSR II System (BD Bioscience), FACSDiva software (BD Bioscience), and FCS Express software (De Novo Software, Los Angeles, CA, USA) were employed to perform a flow cytometry analysis, to acquire and to analyze obtained data, respectively. Rainbow Beads (BD Bioscience) was used to check the day-to-day consistency of measurements. To exclude debris and cell aggregates, viable cells were gated on forward scatter (FSC) and side scatter (SSC). hMSPCs were defined by their phenotype and analyzed on a logarithmic scale. Data from all donors were analyzed by collecting 10,000 events under identical parameters.

\subsection{Multilineage Differentiation Analysis}

A seeding density of hMSPCs was established at $10^{4} \mathrm{cells} / \mathrm{cm}^{2}$, and the cells were seeded in an expansion medium composed of Dulbecco's modified Eagle's medium (DMEM-F12; GIBCO Invitrogen), $10 \%$ FBS (Lonza, Braine-l'Alleud, Belgium), 1\% L-glutamine, 1\% penicilline/streptomycine, and $0.1 \%$ amphotericine B. Additionally, $100 \mathrm{nM}$ dexamethasone, $0.1 \mathrm{mM}$ ascorbic acid-2-phosphate, and 10 $\mathrm{mM} \beta$-glycerophosphate (all Sigma Aldrich, St. Louis, MO, USA) were added to the differentiation medium to induce osteogenesis. Histochemical staining (Alkaline Phosphatase kit No. 85; Sigma Aldrich) was used to assay alkaline phosphatase (ALP) activity after 7 and 14 days of culture. According to the instructions of the manufacturer, ALP enzyme activity was calculated basing on the absorbance of p-nitrophenol phosphate $(405 \mathrm{~nm})$ [26]. Adipogenic differentiation was performed in a medium containing $100 \mathrm{nM}$ dexamethasone, $50 \mu \mathrm{M}$ indomethacine (Sigma Aldrich), and 0.135 IE/mL insulin (Novo Nordisk, Bagsværd, Denmark), and was detected by Oil Red O staining of the adipocyte specific fat vacuoles after 21 days of culture. Chondrogenic differentiation was initiated by culturing cells in DMEM-F12 supplemented with 10\% FBS, $100 \mu \mathrm{M} \mathrm{L}$-ascorbic acid, and $1 \mathrm{ng} / \mathrm{mL}$ TGF- $\beta 3$ (Lonza). Alcian blue staining was applied to verify the production of glycosaminoglycans and mucopolysaccharides after 21 days of culture. Cells were then fixed with $10 \%$ formaldehyde and stained with $1 \%$ Alcian blue in $3 \%$ acetic acid solution at $\mathrm{pH} 2.5$. 


\subsection{Real-Time RT-PCR}

RNeasy Mini Kit and DNase-I treatment (Qiagen, Hilden, Germany) were used to isolate total RNA from undifferentiated and osteogenic differentiated hMSPCs cultured on different GO surface modifications (on the GO-uppermost surface of the composites) on day 21. A total of $1 \mu \mathrm{g}$ of RNA was reverse transcribed with iScriptcDNA Synthesis Kit, (BioRad Laboratories Inc., Hercules, CA, USA) using a blend of oligo(dT) and random hexamer primers. SsoAdvanced Universal SYBR Green Supermix (Bio-Rad) and CFX96 Touch (BioRad) were used for the amplification and measurements, respectively. A standard 3-step PCR temperature protocol (annealing temperature of $60^{\circ} \mathrm{C}$ ) was used for each qPCR, and was followed by a melting curve protocol both to confirm a single gene-specific peak and to detect primer dimerization. $\Delta \Delta \mathrm{Ct}$ method was used for the relative quantification of expression levels, and was based on the geometric mean of the internal controls TBP (TATA-box binding protein), RPLP0 (ribosomal protein, lateral stalk, subunit P0), and B2M ( $\beta-2$ microglobulin), respectively. The expression levels $(\mathrm{C} t)$ of the target genes were normalized to the reference genes $(\Delta \mathrm{Ct})$, and the difference between the $\Delta \mathrm{Ct}$ value of the test sample and the $\Delta \mathrm{Ct}$ of the control sample gave the $\Delta \Delta \mathrm{Ct}$ value. Consequently, the expression ratio was calculated as $2^{\Delta \Delta C t}$. Three QuantiTect primer assays (Qiagen) were selected for real time RT-PCR, namely ALPL, SPARC, and BMP2.

\subsection{Statistical Analysis}

Differences between groups were evaluated by means of a Student's unpaired $t$-test and the exact Wilcoxon test with the PASW statistics 18 software (IBM Corporation, Somers, NY, USA). Two-sided $p$-values $\left(p<0.001^{* * *} ; p<0.01^{* *} ; p<0.05^{*}\right)$ were considered statistically significant. SigmaPlot ${ }^{\circledR} 14.0$ (Systat Software Inc., San Jose, CA, USA) was used to make graphical representations.

\section{Results}

\subsection{Surface Characterization of GO/PMMA}

The protocol of GO paper fabrication resulted in the formation of a self-supporting, uniform, and black material, with the average thickness of $5 \pm 1 \mu \mathrm{m}$ for $\mathrm{GO}_{(\mathrm{A})}$ and $16 \pm 1 \mu \mathrm{m}$ for $\mathrm{GO}_{(\mathrm{B})}$, and the average specific weight of $0.87 \pm 0.08 \mathrm{mg} / \mathrm{cm}^{2}$ for $\mathrm{GO}_{(\mathrm{A})}$ and $2.90 \pm 0.08 \mathrm{mg} / \mathrm{cm}^{2}$ for $\mathrm{GO}_{(\mathrm{B})}$. The morphology of $\mathrm{GO}_{(\mathrm{A})}$ and $\mathrm{GO}_{(\mathrm{B})}$, as presented in the SEM images (Figure 1), showed some wrinkles on the surfaces which were the most probably the edges of graphene oxide, revealing strong adhesion between GO platelets. Overall, the surface of GO paper was relatively smooth, and there were no obvious defects (pores or cracks) observed.
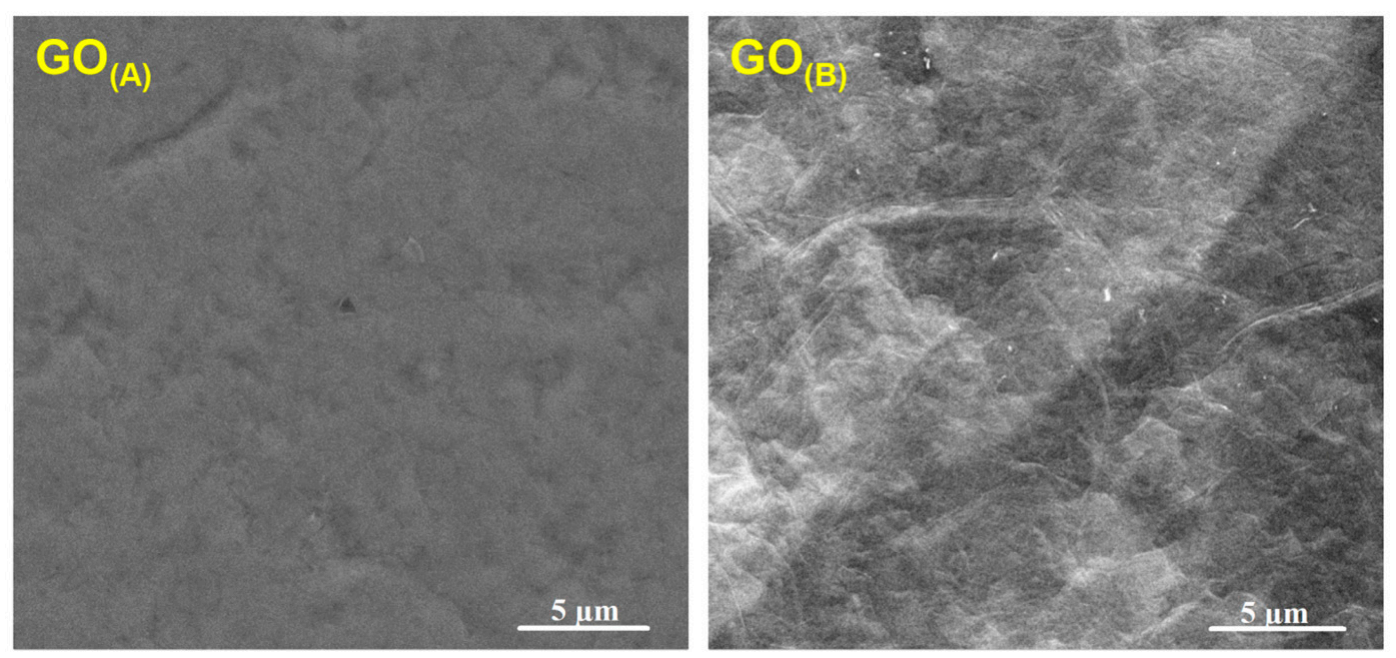

Figure 1. SEM micrographs presenting surface morphology of $\mathrm{GO}_{(\mathrm{A})}$ and $\mathrm{GO}_{(\mathrm{B})}$ paper. 
As demonstrated in SEM micrographs of GO/PMMA composites (Figure 2), PMMA was covering the surface of GO paper, and more uniform surface was obtained when a thin $\mathrm{GO}_{(\mathrm{A})}$ paper was used as a filler. The interface between GO and PMMA can be seen as a border region, particularly in the SEM image of $\mathrm{GO}_{(\mathrm{A})} / \mathrm{PMMA}_{(\mathrm{P})}$. $\mathrm{GO}_{(\mathrm{A})}$ paper was thickly coated with PMMA, while the presence of a thick $\mathrm{GO}_{(B)}$ paper was found to introduce wrinkles to the surface of polymer composite. Moreover, $\mathrm{GO}_{(\mathrm{B})}$ paper seemed to protrude cleanly from the fracture site. The surface of $\mathrm{GO}_{(\mathrm{B})} / \mathrm{PMMA}_{(\mathrm{M})}$ was observed to exhibit a significant number of voids and pores.

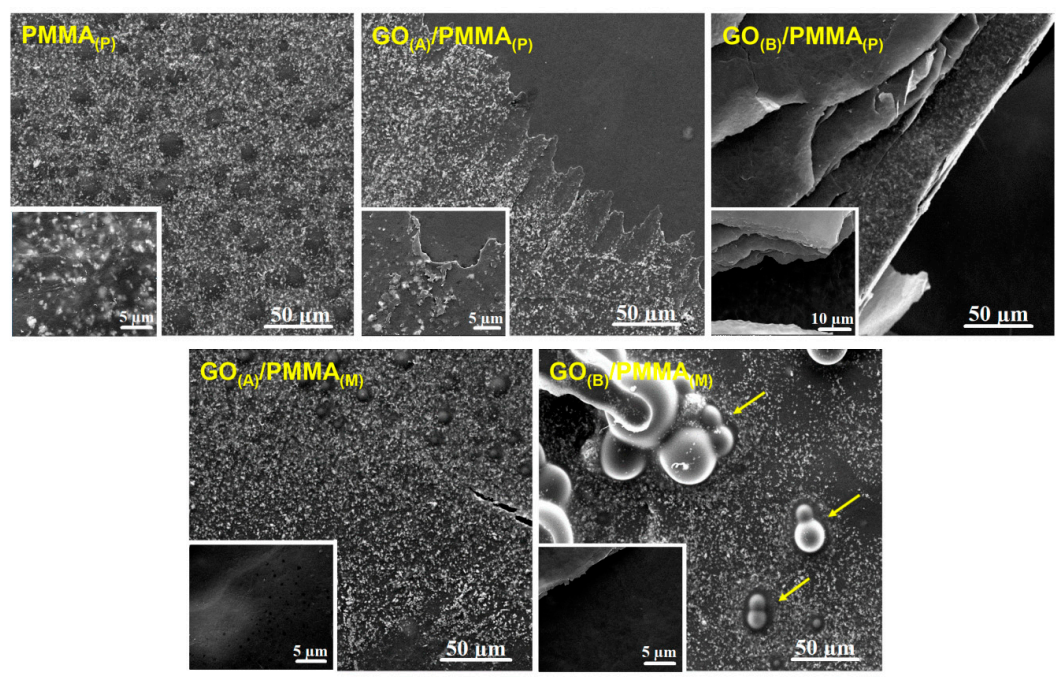

Figure 2. SEM micrographs presenting surface morphology of $\mathrm{GO}_{(\mathrm{A})} / \mathrm{PMMA}_{(\mathrm{P})}, \mathrm{GO}_{(\mathrm{B})} / \mathrm{PMMA}_{(\mathrm{P})}$, $\mathrm{GO}_{(\mathrm{A})} / \mathrm{PMMA}_{(\mathrm{M})}$, and $\mathrm{GO}_{(\mathrm{B})} / \mathrm{PMMA}_{(\mathrm{M})}$ (arrows indicate discussed surface structures), as well as untreated PMMA, with high magnification images as the insets.

\section{2. hMSPC Characterization and Multilineage Differentiation Analysis}

Cells providing morphologic characteristics of human primary MSPCs (mononuclear, fibroblast-like, spindle shaped, plastic-adherent) were isolated from all samples within 4-8 days. hMSPCs showed a positive expression of CD73 (99.8 $\pm 0.1 \%)$, CD90 (99.9 $\pm 0.1 \%)$, CD105 $(69.1 \pm 9.8 \%)$ of gated cells. The typical forward/side scatter characteristics of $71.5 \pm 4.9 \%$ were gated. The negativity for CD14 (0.2 $\pm 0.2 \%)$, CD19 (0.6 $\pm 0.1 \%)$, CD34 $(0.4 \pm 0.3 \%)$, CD45 (23.9 $\pm 7.8 \%)$, and HLA-DR $(0.5 \pm 0.3 \%)$ confirmed the phenotype of MSPCs (Figure $3 \mathrm{~A})$.

ALP activity was measured of absorbance (optical dense, OD) of p-nitrophenol in supernatant at the wavelength of $405 \mathrm{~nm}$ over 14 days (Figure 3B). ALP expression was detected on day 7 and day 14 , respectively, when the cells were osteogenically differentiated with a significant increase $(p<0.001)$. No expression of ALP was observed in any of the samples of undifferentiated negative controls. Due to the interaction of the cationic dye Alcian blue and acid glycosaminoglycans, augmented blue coloration was noticed for chondrogenic differentiated hMSPCs, and not for undifferentiated controls (Figure 3C). As a result of chondrogenic differentiation, a 4.7-fold increase $(p<0.05)$ was noticed for the expression of aggrecan. In order to demonstrate the multilineage ability, hMSPCs were also differentiated in the adipogenic lineage. The adipogenic cell differentiation was demonstrated with the formation of lipid vacuoles which were visualized by Oil Red O staining on day 21 (Figure 3D). These results explicitly characterized our primary cells as hMSCPs. 
A
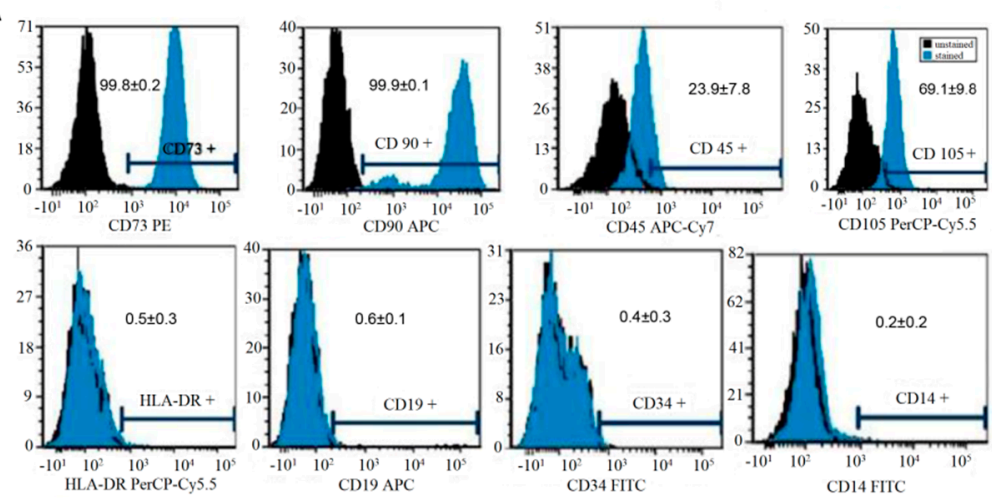

B
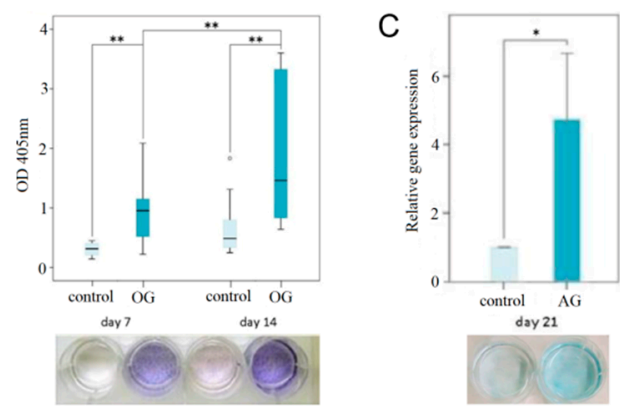

$\mathrm{D}$

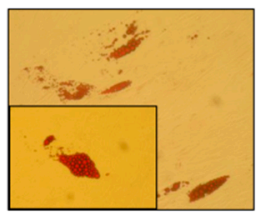

Figure 3. hMSPC characterization and multilineage differentiation analysis. The used hMSPCs were characterized according (A) the positive expression of CD73, CD90, CD105, and negative expression CD14, CD19, CD34, CD45, and HLA-DR using multicolor fluorescence-activated cell sorting analyses. The values indicated the percentage of positively stained cells. The capacity for multilineage differentiation potential was confirmed by (B) ALP staining for osteogenic differentiation, (C) Alcian blue staining and the expression of aggrecan for the chondrogenic differentiation, and (D) the Oil Red O staining of lipid droplets for the adipogenic lineage; $p<0.01^{* *} ; p<0.05^{*}$ ).

\subsection{Efficiency of Osteogenic Differentiation}

ALP, SPARC, and BMP-2 assays were performed to assess the mineralization of hMSPCs cultured on the surface of $\mathrm{GO}_{(\mathrm{A})} / \mathrm{PMMA}_{(\mathrm{P})}, \mathrm{GO}_{(\mathrm{B})} / \mathrm{PMMA}_{(\mathrm{P})}, \mathrm{GO}_{(\mathrm{A})} / \mathrm{PMMA}_{(\mathrm{M})}$ and $\mathrm{GO}_{(\mathrm{B})} / \mathrm{PMMA}_{(\mathrm{M})}$, as well as untreated PMMA. Consequently, the results shown in Figure 4 describe how strongly the expression of individual markers was increased by the osteogenic differentiation medium, with the undifferentiated hMSPCs as the control. As demonstrated by ALP assay, all investigated surfaces led to a significant increase in mineral deposition, with the most pronounced effect noticed for $\mathrm{GO}_{(\mathrm{B})} / \mathrm{PMMA}_{(\mathrm{M})}$ (8-fold increase when compared with a control). The same material was also found to lead to the significant increase in the expression of SPARC (2-fold increase with respect to control), even though the highest relative gene expression (3-fold increase with respect to control) was noticed for unmodified PMMA. All investigated surfaces, including GO/PMMA composites as well as unmodified PMMA samples, were shown to decrease the relative BMP-2 expression from 7 to 9 times when compared with a control.

The relative gene expression profiles of hMSPCs cultured in normal expansion medium were analyzed with respect to ALP, SPARC and BMP-2, and compared with an unmodified PMMA as the control. Consequently, the results presented in Figure 5 describe the expression of the individual markers by the osteogenic differentiation medium in relation to an unmodified PMMA control These results showed the unchanged SPARC expression, simultaneously with the decrease in ALP expression (approximately 2-fold) and a significant increase of BMP-2 expression (from 2-fold for $\mathrm{GO}_{(\mathrm{A})} / \mathrm{PMMA}_{(\mathrm{P})}$ to 2.5-fold for $\left.\mathrm{GO}_{(\mathrm{A})} / \mathrm{PMMA}_{(\mathrm{M})}\right)$ for $\mathrm{GO} / \mathrm{PMMA}$ composites with respect to a PMMA control. 

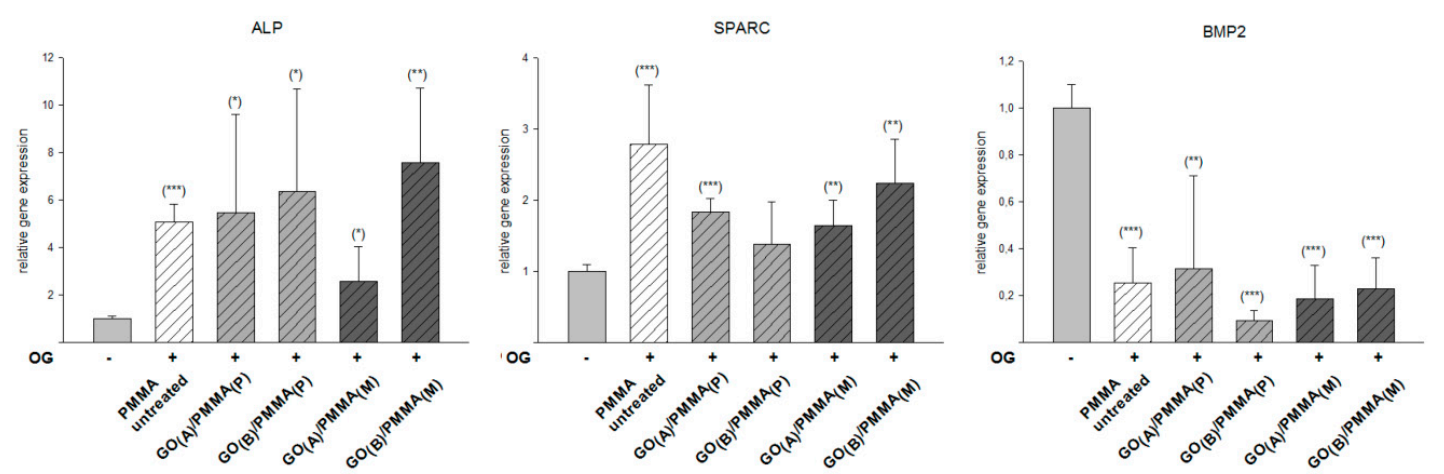

Figure 4. The expression of specific osteogenic markers (ALP, SPARC, and BMP-2) of hMSPCs cultured in the presence of osteogenic differentiation medium for 21 days on the surface of GO/PMMA composites as well as untreated PMMA, compared with undifferentiated hMSPCs cultivated in expansion medium as the negative control; $p<0.001^{* *} ; p<0.01^{* *} ; p<0.05^{*}$.
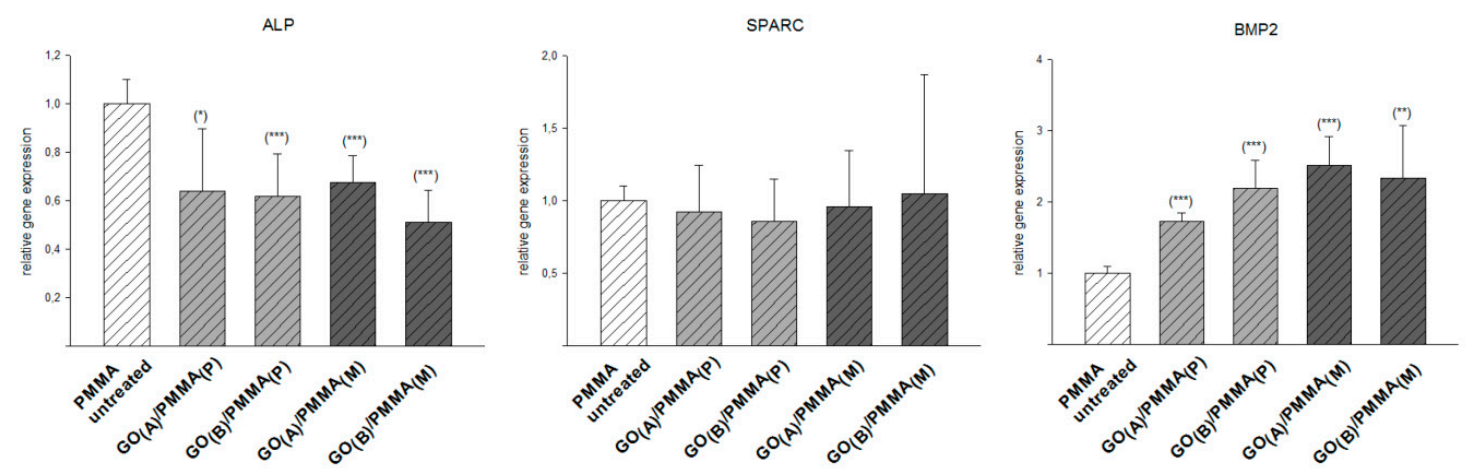

Figure 5. The expression of specific osteogenic markers (ALP, SPARC, and BMP-2) of hMSPCs cultured in the absence of a specific induction medium for 21 days on the surface of GO/PMMA composites as well as untreated PMMA; $p<0.001^{* * *} ; p<0.01^{* *} ; p<0.05^{*}$.

To estimate the efficiency of osteogenic differentiation, GO/PMMA coatings were compared with an unmodified PMMA as a control (Figure 6). This kind of evaluation was chosen to show the efficiency of the osteogenic differentiation ability of each group in relation to the PMMA control. Particularly, $\mathrm{GO}_{(\mathrm{B})} / \mathrm{PMMA}_{(\mathrm{M})}$ composite was found to improve the expression of osteogenic differentiation markers (2-fold increase for ALP, 1.5-fold increase for SPARC, and 2-fold increase for BMP-2), while $\mathrm{GO}_{(\mathrm{A})} / \mathrm{PMMA}_{(\mathrm{M})}$ was found to decrease ALP expression (2-fold). All other effects were assessed as not significant.
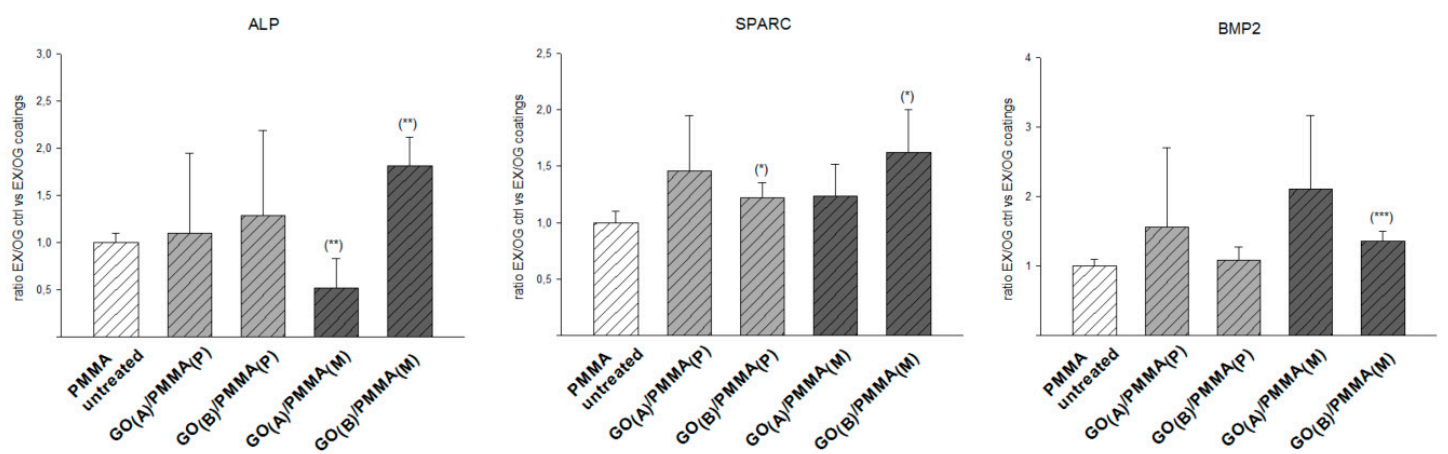

Figure 6. The expression of specific osteogenic markers (ALP, SPARC, and BMP-2) of hMSPCs cultured for 21 days on the surface of GO/PMMA composites with respect to a PMMA control; $p<0.001^{* * *}$; $p<0.01^{* *} ; p<0.05^{*}$. 


\section{Discussion}

The greatest challenge of modern nanotechnology is to expand its scope from nanoscale into macroscale. Therefore, the possibility of fabricating free-standing, paper-like materials basing on nanoscale components is a subject of intensive research [27]. The protocol of GO paper fabrication, introduced by us, allowed formation of self-supporting, uniform films composed of stacked platelets of graphene oxide. The irregularity of surface of GO paper seemed to increase with its thickness, which is of special importance for further biological studies since higher roughness promotes cell adhesion [28].

GO paper is known to possess many functional groups, and could easily form hydrogen bonds with hydrophilic polymers. The presence of hydrophobic methacrylate groups in PMMA, however, discourages these interactions to occur. Therefore, since PMMA chains would rather remain in a coiled conformation, they are supposed to fit well into wavy structures of the nanosheets of GO paper leading to more efficient packing within intersheet gallery [29]. Consequently, SEM micrographs demonstrated a good interlocking between GO paper and PMMA matrix. Particularly $\mathrm{GO}_{(\mathrm{A})}$ paper was found to be thickly coated with PMMA, suggesting strong adhesion between this polymer and $\mathrm{GO} . \mathrm{GO}_{(\mathrm{B})}$ paper, on the other hand, seemed to protrude from the fracture site, suggesting a weak interfacial bonding between $\mathrm{GO}_{(\mathrm{B})}$ and PMMA. This is consistent with some previous studies indicating that small sizes of GO sheets promote the formation of homogeneous composites [30]. Moreover, the presence of voids and pores on the surface of $\mathrm{GO}_{(\mathrm{B})} / \mathrm{PMMA}_{(\mathrm{M})}$ could be associated with the presence of unreacted residual monomer, which is volatile and is supposed to be released after polymerization [31]. This would mean that some double bonds present in GO paper could be attacked by the radical species formed during MMA polymerization, retarding or inhibiting the reaction of polymerization [24].

As a biological model, cells providing morphologic characteristics of human primary MSPCs (mononuclear, fibroblast-like, spindle shaped, plastic-adherent) were isolated and cultured on the surface of GO/PMMA composites. The phenotype of hMSPCs was confirmed according to the criteria of the International Society for Cellular Therapy [32] for defining multipotent mesenchymal stromal cells. In addition, hMSPCs were successfully differentiated towards the osteogenic, chondrogenic, and adipogenic lineage, which was confirmed by ALP expression, Alcian blue staining, aggrecan expression, and Oil Red $\mathrm{O}$ staining. To assess the efficiency of osteogenic differentiation of hMSPCs cultured onto PMMA as well as GO/PMMA composites, three differentiation markers were analyzed, namely alkaline phosphatase (ALP), secreted protein acidic and rich in cysteine (SPARC), and bone morphogenetic protein-2 (BMP-2). ALP is a metalloenzyme playing an important role in the mineralization of tissue cells [33]. ALP is found to act as both a mineralization promoter by increasing the local concentration of phosphate, as well as an inhibitor of mineral formation by decreasing the concentration of extracellular pyrophosphate. Since ALP is observed to be highly expressed in mineralized cells, it can be used to predict their bone forming capacity under different conditions. Osteonectin (SPARC), on the other hand, is the most abundant non-collagenous extracellular matrix protein present in bone [34]. SPARC gene dosage has a dramatic effect on bone volume and is one of critical regulators of bone remodeling, calcium turnover and an initiator of mineralization [34,35]. Therefore, SPARC can be used for the examination of osteogenic differentiation. Another marker for osteogenic differentiation is bone morphogenetic protein-2 (BMP-2), which is known as a potent osteogenic factor with roles in both normal bone healing and pathological bone formation in soft tissues [36]. BMP-2 is found to facilitate osteogenic differentiation through inducing ALP activity, promoting mineralization and enhancing adherence of cells [37].

As demonstrated by ALP assay, all investigated surfaces led to a significant increase in mineral deposition. This effect was accompanied with a decrease in BMP-2 expression, which might suggest that the presence of a differentiation medium had a stronger effect on the osteogenic differentiation than BMP-2, resulting in down regulation of the latter. Nevertheless, the expression of specific osteogenic markers, such as ALP and SPARC, was found to be significantly increased by the osteogenic differentiation medium. In the absence of a specific induction medium, on the other hand, the cells cultured on GO/PMMA composites were found to be able to induce an increase in BMP-2. In this way, 
GO/PMMA composites were shown to be able to drive cellular differentiation without any addition of osteogenic supplements, just as reported for hMSCs in collagen matrices subjected cyclic tensile strain [38], even though the process of osteogenic differentiation was found to be much more effective when cells were culture in the presence of a medium trigger.

The comparison of GO/PMMA coatings with an unmodified PMMA as a control suggested that among all investigated GO/PMMA composite materials, $\mathrm{GO}_{(\mathrm{B})} / \mathrm{PMMA}_{(\mathrm{M})}$ composite was the most efficient inducer of osteogenic differentiation, particularly basing on the relative expression of ALP (other changes were found to be statistically non-significant). This effect should be assigned to its surface morphology, as exhibiting a significant number of voids and pores. As presented by Abagnale et al. [39], specific patterns present on the surface can boost differentiation of MSCs towards specific cell types. These patterns should be in the micrometer range to be able to support the differentiation processes initiated by induction media, and this requirement is met particularly by $\mathrm{GO}_{(\mathrm{B})}$ paper and $\mathrm{GO}_{(\mathrm{B})} / \mathrm{PMMA}_{(\mathrm{M})}$ composite. All these data suggest that GO/PMMA composites, particularly $\mathrm{GO}_{(\mathrm{B})} / \mathrm{PMMA}_{(\mathrm{M})}$ produced manually with a thick GO paper, may direct hMSPCs toward osteogenic differentiation and can serve as promising materials in bone tissue engineering. The properties of GO/PMMA make this material advantageous for potential applications as screws or implants fixation. Previous studies [25,40] showed that PMMA filled with GO conforms to physicochemical and mechanical demands of these clinical applications. In this study, we have shown that incorporation of GO into PMMA matrix may provide an additional functionality to the resulting composite material, enhancing its biocompatibility through facilitating osteogenesis. Still, before the introduced material could be considered as a bone cement, further studies should include a comprehensive biomechanical characterization of GO/PMMA.

\section{Conclusions}

In this paper, we present the potency of GO/PMMA composites to induce osteogenic differentiation of hMSPCs. Through the analysis of three differentiation markers, namely ALP, SPARC, and BMP-2 in the presence and in the absence of a specific induction medium, we were able to assess the efficiency of osteogenic differentiation of hMSPCs cultured on four types of GO/PMMA composites, differing in the thickness of GO paper as well as the method of fabrication of the composite. All investigated GO/PMMA composite materials were found to effectively induce osteogenic differentiation, and to outperform both unmodified PMMA and a negative control (undifferentiated hMSPCs). Among GO/PMMA composite materials, a composite produced manually with a thick GO paper $\left(\mathrm{GO}_{(\mathrm{B})} / \mathrm{PMMA}_{(\mathrm{M})}\right)$ acted as the most efficient inducer of osteogenic differentiation, particularly basing on the relative expression of ALP (other changes were found to be statistically non-significant). Since $\mathrm{GO}_{(\mathrm{B})} / \mathrm{PMMA}_{(\mathrm{M})}$ was the composite surface possessing a significant number of voids and pores, its developed surface morphology was supposed to be responsible for directing hMSPCs toward osteogenic differentiation. In this way, GO/PMMA composites, and particularly $\mathrm{GO}_{(\mathrm{B})} / \mathrm{PMMA}_{(\mathrm{M})}$, were shown as promising materials in bone tissue engineering.

Author Contributions: Conceptualization, D.P., B.L., and F.A.; Investigation, D.P., N.S., B.L., and F.A.; Methodology, D.P., B.L., and F.A.; Supervision, F.A.; Writing-original draft, K.K.; Writing-review and editing, D.P., B.L., and F.A. All authors have read and agreed to the published version of the manuscript.

Funding: The cell studies described in this work were supported by the FFG Bridge program (Grant No. 861608). K.K. would like to thank the National Science Center, Poland (SONATA program, Grant No. 2016/23/D/ST5/01306) and the Silesian University of Technology, Poland (04/040/BK_20/0113).

Acknowledgments: The authors thank Sandra Ngo and Mariangela Fedel for their support in fabricating the GO, and Heike Kaltenegger for her support in isolating and characterizing the primary hMSPCs.

Conflicts of Interest: The authors declare no conflict of interest. 


\section{References}

1. Novoselov, K.S.; Geim, A.K.; Morozov, S.V.; Jiang, D.; Zhang, Y.; Dubonos, S.V.; Grigorieva, I.V.; Firsov, A.A. Electric field in atomically thin carbon films. Science 2004, 306, 666-669. [CrossRef] [PubMed]

2. Ghosh, S.; Calizo, I.; Teweldebrhan, D.; Pokatilov, E.P.; Nika, D.L.; Balandin, A.A.; Bao, W.; Miao, F.; Lau, C.N. Extremely high thermal conductivity of graphene: Prospects for thermal management applications in nanoelectronic circuits. Appl. Phys. Lett. 2008. [CrossRef]

3. Lee, J.; Kim, J.; Kim, S.; Min, D.H. Biosensors based on graphene oxide and its biomedical application. Adv. Drug Deliv. Rev. 2016, 105, 275-287. [CrossRef] [PubMed]

4. Chen, H.; Müller, M.B.; Gilmore, K.J.; Wallace, G.G.; Li, D. Mechanically strong, electrically conductive, and biocompatible graphene paper. Adv. Mater. 2008, 20, 3557-3561. [CrossRef]

5. Chung, C.; Kim, Y.K.; Shin, D.; Ryoo, S.R.; Hong, B.H.; Min, D.H. Biomedical applications of graphene and graphene oxide. Acc. Chem. Res. 2013, 46, 2211-2224. [CrossRef]

6. Liu, J.; Cui, L.; Losic, D. Graphene and graphene oxide as new nanocarriers for drug delivery applications. Acta Biomater. 2013, 9, 9243-9257. [CrossRef]

7. Choudhary, P.; Parandhaman, T.; Ramalingam, B.; Duraipandy, N.; Kiran, M.S.; Das, S.K. Fabrication of Nontoxic Reduced Graphene Oxide Protein Nanoframework as Sustained Antimicrobial Coating for Biomedical Application. ACS Appl. Mater. Interfaces 2017, 9, 38255-38269. [CrossRef]

8. Liu, Q.; Wei, L.; Wang, J.; Peng, F.; Luo, D.; Cui, R.; Niu, Y.; Qin, X.; Liu, Y.; Sun, H.; et al. Cell imaging by graphene oxide based on surface enhanced Raman scattering. Nanoscale 2012, 4, 7084-7089. [CrossRef]

9. Yin, F.; Hu, K.; Chen, Y.; Yu, M.; Wang, D.; Wang, Q.; Yong, K.T.; Lu, F.; Liang, Y.; Li, Z. SiRNA delivery with PEGylated graphene oxide nan osheets for combined photothermal and genetherapy for pancreatic cancer. Theranostics 2017, 7, 1133-1148. [CrossRef]

10. Wan, C.; Chen, B. Poly( $\varepsilon$-caprolactone)/graphene oxide biocomposites: Mechanical properties and bioactivity. Biomed. Mater. 2011, 6, 055010. [CrossRef]

11. Baradaran, S.; Moghaddam, E.; Basirun, W.J.; Mehrali, M.; Sookhakian, M.; Hamdi, M.; Moghaddam, M.R.N.; Alias, Y. Mechanical properties and biomedical applications of a nanotube hydroxyapatite-reduced graphene oxide composite. Carbon N. Y. 2014, 69, 32-45. [CrossRef]

12. Xie, C.; Lu, X.; Han, L.; Xu, J.; Wang, Z.; Jiang, L.; Wang, K.; Zhang, H.; Ren, F.; Tang, Y. Biomimetic Mineralized Hierarchical Graphene Oxide/Chitosan Scaffolds with Adsorbability for Immobilization of Nanoparticles for Biomedical Applications. ACS Appl. Mater. Interfaces 2016, 8, 1707-1717. [CrossRef] [PubMed]

13. Bai, H.; Li, C.; Wang, X.; Shi, G. A pH-sensitive graphene oxide composite hydrogel. Chem. Commun. 2010, 46, 2376-2378. [CrossRef] [PubMed]

14. Thampi, S.; Muthuvijayan, V.; Parameswaran, R. Mechanical characterization of high-performance graphene oxide incorporated aligned fibroporous poly(carbonate urethane) membrane for potential biomedical applications. J. Appl. Polym. Sci. 2015, 132, 41809. [CrossRef]

15. Byun, E.; Lee, H. Enhanced loading efficiency and sustained release of doxorubicin from hyaluronic acid/graphene oxide composite hydrogels by a mussel-inspired catecholamine. J. Nanosci. Nanotechnol. 2014, 16, 7395-7401. [CrossRef]

16. Shen, J.; Yan, B.; Li, T.; Long, Y.; Li, N.; Ye, M. Mechanical, thermal and swelling properties of poly(acrylic acid)-graphene oxide composite hydrogels. Soft Matter 2012, 8, 1831-1836. [CrossRef]

17. Feuser, P.E.; Gaspar, P.C.; Ricci-Júnior, E.; Da Silva, M.C.S.; Nele, M.; Sayer, C.; De Araújo, P.H.H. Synthesis and characterization of poly(methyl methacrylate) pmma and evaluation of cytotoxicity for biomedical application. Macromol. Symp. 2014, 343, 65-69. [CrossRef]

18. Frazer, R.Q.; Byron, R.T.; Osborne, P.B.; West, K.P. PMMA: An essential material in medicine and dentistry. J. Long-Term. Eff. Med. Implants 2005, 15, 629-639. [CrossRef]

19. Gohil, S.V.; Suhail, S.; Rose, J.; Vella, T.; Nair, L.S. Polymers and Composites for Orthopedic Applications. In Materials and Devices for Bone Disorders; Bose, S., Bandyopadhyay, A., Eds.; Elsevier/Academic Press: Amsterdam, The Netherlands, 2017; ISBN 9780128028032.

20. He, Q.; Chen, H.; Huang, L.; Dong, J.; Guo, D.; Mao, M.; Kong, L.; Li, Y.; Wu, Z.; Lei, W. Porous surface modified bioactive bone cement for enhanced bone bonding. PLoS ONE 2012, 7, e42525. [CrossRef] 
21. Miola, M.; Bistolfi, A.; Valsania, M.C.; Bianco, C.; Fucale, G.; Verné, E. Antibiotic-loaded acrylic bone cements: An in vitro study on the release mechanism and its efficacy. Mater. Sci. Eng. C 2013, 33, 3025-3032. [CrossRef]

22. Ormsby, R.W.; Modreanu, M.; Mitchell, C.A.; Dunne, N.J. Carboxyl functionalised MWCNT/polymethyl methacrylate bone cement for orthopaedic applications. J. Biomater. Appl. 2014, 29, 209-221. [CrossRef] [PubMed]

23. Fang, C.H.; Lin, Y.W.; Sun, J.S.; Lin, F.H. The chitosan/tri-calcium phosphate bio-composite bone cement promotes better osteo-integration: An in vitro and in vivo study. J. Orthop. Surg. Res. 2019, 14, 162. [CrossRef] [PubMed]

24. Paz, E.; Forriol, F.; Del Real, J.C.; Dunne, N. Graphene oxide versus graphene for optimisation of PMMA bone cement for orthopaedic applications. Mater. Sci. Eng. C 2017, 77, 1003-1011. [CrossRef]

25. Paz, E.; Ballesteros, Y.; Abenojar, J.; Del Real, J.C.; Dunne, N.J. Graphene oxide and graphene reinforced PMMA bone cements: Evaluation of thermal properties and biocompatibility. Materials 2019, 12, 3146. [CrossRef] [PubMed]

26. Sabokbar, A.; Millett, P.J.; Myer, B.; Rushton, N. A rapid, quantitative assay for measuring alkaline phosphatase activity in osteoblastic cells in vitro. Bone Miner. 1994, 27, 57-67. [CrossRef]

27. Dikin, D.A.; Stankovich, S.; Zimney, E.J.; Piner, R.D.; Dommett, G.H.B.; Evmenenko, G.; Nguyen, S.T.; Ruoff, R.S. Preparation and characterization of graphene oxide paper. Nature 2007, 448, 457-460. [CrossRef]

28. Ku, S.H.; Park, C.B. Myoblast differentiation on graphene oxide. Biomaterials 2013, 34, 2017-2023. [CrossRef]

29. Compton, O.C.; Putz, K.W.; Brinson, L.C.; Nguyen, S.T. Composite Graphene Oxide-Polymer Laminate and Method. U.S. Patent Application No. 8,709,213, 20 October 2011.

30. Gudarzi, M.M.; Sharif, F. Self assembly of graphene oxide at the liquid-liquid interface: A new route to the fabrication of graphene based composites. Soft Matter 2011, 7, 3432-3440. [CrossRef]

31. Gonalves, G.; Cruz, S.M.A.; Ramalho, A.; Grácio, J.; Marques, P.A.A.P. Graphene oxide versus functionalized carbon nanotubes as a reinforcing agent in a PMMA/HA bone cement. Nanoscale 2012, 4, 2937-2945. [CrossRef]

32. Dominici, M.; Le Blanc, K.; Mueller, I.; Slaper-Cortenbach, I.; Marini, F.C.; Krause, D.S.; Deans, R.J.; Keating, A.; Prockop, D.J.; Horwitz, E.M. Minimal criteria for defining multipotent mesenchymal stromal cells. The International Society for Cellular Therapy position statement. Cytotherapy 2006, 8, 315-317. [CrossRef]

33. Golub, E.E.; Boesze-Battaglia, K. The role of alkaline phosphatase in mineralization. Curr. Opin. Orthop. 2007, 18, 444-448. [CrossRef]

34. Delany, A.M.; Hankenson, K.D. Thrombospondin-2 and SPARC/osteonectin are critical regulators of bone remodeling. J. Cell Commun. Signal. 2009, 3, 227-238. [CrossRef] [PubMed]

35. Zong, S.; Zeng, G.; Zou, B.; Li, K.; Fang, Y.; Lu, L.; Xiao, D.; Zhang, Z. Effects of Polygonatum sibiricum polysaccharide on the osteogenic differentiation of bone mesenchymal stem cells in mice. Int. J. Clin. Exp. Pathol. 2015, 8, 6169-6180. [PubMed]

36. Rui, Y.F.; Lui, P.P.Y.; Lee, W.Y.W.; Chan, K.M. Higher BMP receptor expression and BMP-2-induced osteogenic differentiation in tendon-derived stem cells compared with bone-marrow-derived mesenchymal stem cells. Int. Orthop. 2012, 36, 1099-1107. [CrossRef] [PubMed]

37. Sun, J.; Li, J.; Li, C.; Yu, Y. Role of bone morphogenetic protein-2 in osteogenic differentiation of mesenchymal stem cells. Mol. Med. Rep. 2015, 12, 4230-4237. [CrossRef]

38. Sumanasinghe, R.D.; Bernacki, S.H.; Loboa, E.G. Osteogenic differentiation of human mesenchymal stem cells in collagen matrices: Effect of uniaxial cyclic tensile strain on bone morphogenetic protein (BMP-2) mRNA expression. Tissue Eng. 2006, 12, 3459-3465. [CrossRef] [PubMed]

39. Abagnale, G.; Steger, M.; Nguyen, V.H.; Hersch, N.; Sechi, A.; Joussen, S.; Denecke, B.; Merkel, R.; Hoffmann, B.; Dreser, A.; et al. Surface topography enhances differentiation of mesenchymal stem cells towards osteogenic and adipogenic lineages. Biomaterials 2015, 61, 316-326. [CrossRef]

40. Park, C.; Park, S.; Lee, D.; Choi, K.S.; Lim, H.P.; Kim, J. Graphene as an Enabling Strategy for Dental Implant and Tissue Regeneration. Tissue Eng. Regen. Med. 2017, 14, 481-493. [CrossRef]

(C) 2020 by the authors. Licensee MDPI, Basel, Switzerland. This article is an open access article distributed under the terms and conditions of the Creative Commons Attribution (CC BY) license (http://creativecommons.org/licenses/by/4.0/). 